www.jmscr.igmpublication.org Impact Factor 5.244

Index Copernicus Value: 83.27

ISSN (e)-2347-176x ISSN (p) 2455-0450

crossref DOI: _https://dx.doi.org/10.18535/jmscr/v4i10.117

\title{
Causative Organisms and Outcome in Patients with Acute Exacerbations of Chronic Obstructive Pulmonary Disease
}

\author{
Authors \\ Dr Smitha Nair P.G ${ }^{1}$, Dr Achuthan .. ${ }^{2}$, Dr Manoj.D.K ${ }^{3}$, Dr Rajani.M ${ }^{4}$, \\ Dr Niyas Naseer ${ }^{5}$, Dr Neethu Thambi ${ }^{6}$
}

${ }^{1}$ Junior Resident Dept of Respiratory Medicine,Pariyaram Medical College, Pariyaram, Kannur, Kerala.

${ }^{2}$ Professor \& HOD, Dept of Respiratory Medicine,Pariyaram Medical College, Pariyaram, Kannur, Kerala

${ }^{3}$ Professor, Dept of Respiratory Medicine, Pariyaram Medical College, Pariyaram,Kannur,Kerala

${ }^{4}$ Professor, Dept of Respiratory Medicine, Pariyaram Medical College, Pariyaram,Kannur,Kerala

${ }^{5}$ Junior Resident Dept of Respiratory Medicine, Pariyaram Medical College, Pariyaram, Kannur, Kerala

${ }^{6}$ Junior Resident, Dept of Respiratory Medicine,Pariyaram Medical College, Pariyaram, Kannur, Kerala Correspondence Author

Dr Rajani M

Professor, Department of Respiratory Medicine, Pariyaram Medical College, Pariyaram, Kannur, Kerala Email: Sajeevanrajani@gmail.com

\section{ABSTRACT}

BACKGROUND: Chronic obstructive pulmonary disease and its exacerbations are a major disease problem in Malabar area of Kerala associated with significant morbidity and mortality.

AIMS AND OBJECTIVES: 1) To identify the causative organisms responsible for the infective exacerbations of chronic obstructive pulmonary disease. 2) To assess the clinical response to antimicrobial therapy based on sputum culture and sensitivity report. 3)To assess the influence of comorbid conditions in the outcome of chronic obstructive pulmonary disease

MATERIALS AND METHODS: A hospital based, non interventional, prospective, study was conducted in the department of respiratory medicine, Pariyaram Medical College, Pariyaram, Kannur, Kerala, India. Study population was 100 consecutive adult COPD patients of either sex. Early morning sputum samples were collected in a sterile container and sent to microbiology department for Gram staining, Culture and sensitivity and acid fast bacilli staining.

RESULTS: Among the total 100 patients studied most common age group was 60-69 years (42\%). The mean age of study population was 65.6yrs.In this study, males constituted $97 \%$ and the rest of them were females. Sputum culture and sensitivity yielded bacterial growth in 67\% of study sample.8\% patients with fungal growth.25\% yielded no growth. Out of 67\% of study sample, 29 were streptococcus pneumoniae, which was the most common organism. 15 were Hemophilus influenza, 6 were Moraxella catarrhalis and 6 were Klebsiella pneumonia.

CONCLUSION: In this study, most of the acute exacerbations of COPD were caused by bacterial infections. The most common organisms isolated were streptococcus pneumoniae.

KEYWORDS: Chronic Obstructive Pulmonary Disease, Sputum Culture And Sensitivity. 


\section{INTRODUCTION}

Chronic obstructive pulmonary disease(COPD) is a leading cause of morbidity and mortality worldwide. Global Burden of Disease study projected that chronic obstructive pulmonary disease which ranked sixth as a cause of death in 1990, will become the third leading cause of death worldwide by 2020 , a latest projection estimated it to be the fourth leading cause of death by year $2030^{1}$. According to the estimate of Global Burden of Disease Study, by the year 2020, COPD is likely to become the fifth leading cause of disability adjusted life years (DALYS). Chronic obstructive pulmonary disease is characterized by the progressive development of COPD affects more than five percent of the population and is associated with high morbidity and mortality ${ }^{3}$.It is the third ranked cause of death in the United States, killing more than 1, 20,000 individual each year. ${ }^{4}$

COPD is more in countries where smoking is highly prevalent. Working population in occupations associated with high levels of particulates in the inspired air and women in underdeveloped countries with long term exposure to indoor open fires for cooking and heating are examples of groups who are prone to COPD without smoking cigarettes.

Acute exacerbations of chronic obstructive pulmonary disease present as a worsening of the previous stable state and include some, or all of such clinical features as increased dyspnea, wheeze, cough, sputum volume, the presence or the development of airflow limitation that is not fully reversible. Exacerbations and comorbidities contribute to the overall severity in individual patients. ${ }^{2}$

Chronic obstructive pulmonary disease and its exacerbations are a major disease problem in Malabar area of Kerala associated with significant morbidity and mortality. No specific data is available regarding the spectrum of organisms and influence of co-morbidities in the exacerbation of COPD.

\section{AIMS AND OBJECTIVES}

1. To identify the causative bacteria's responsible for the infective exacerbations of chronic obstructive pulmonary disease.

2. To assess the clinical response to antimicrobial therapy based on sputum culture and sensitivity report.

3. To assess the influence of comorbid conditions in the outcome of chronic obstructive pulmonary disease.

\section{MATERIALS AND METHODS}

A hospital based, non interventional, prospective, study was conducted in the department of respiratory medicine, Pariyaram Medical College, Pariyaram, Kannur, Kerala, India. Study population was 100 consecutive adult patients of either sex. Inclusion Criteria were Patients with COPD admitted with one or more of the symptoms like Increased dyspnea, Increased sputum production \& Increased sputum purulence. Exclusion criteria were patients below the age of 40,patients with other lung disease, (Chest X-ray evidence of other causes of obstructive lung disease.), patients who are unwilling \& patients with congestive cardiac failure. In each case, an informed consent was taken. Patients name, age and occupational history will be noted.

Presenting complaints and history of presenting complaints were enquired into. Past medical and surgical history, personal history including smoking history was included.

Co-morbid conditions like diabetes mellitus, hypertension and ischemic heart diseases were noted. General and systemic examinations were done. Investigations like routine blood and urine examinations and other relevant investigations were done.

Early morning sputum samples were collected in a sterile container and sent to microbiology department for Gram staining, Culture and sensitivity and acid fast bacilli staining. X-ray chest and ECG were taken. Chest radiographs were useful in excluding alternative diagnosis. An ECG may aid in the diagnosis of coexisting cardiac problems with Echocardiogram studies in 
relevant cases. Patients were started on empirical antibiotic therapy after sputum collection and antibiotics changed after the sputum culture and sensitivity report. Patient's prognoses were noted on regular basis. Pulmonary function test were done once the general condition of the patient improves.

\section{STATISTICAL PROCEDURE}

The collected data were entered and analyzed using statistical software electronically

\section{RESULTS}

Among the total 100 patients studied most common age group was 60-69 years (42\%). The mean age of study population was 65.6yrs.In this study, males constituted $97 \%$ and the rest of them were females. $53 \%$ of the total study samples were unskilled workers. All patients had breathlessness \& cough. 94 patients had increased sputum purulence.47 patients had fever. Majority of the patients (32) were having Diabetes mellitus. Coronary artery disease was present in 29 patients. Hypertension was present in 26 patients.13 patients did not have any associated co-morbid illness. The initial Chest X-ray of 100 patients studied at the time of admission, 47 patients showed parenchymal infiltrates apart from COPD changes All patients were sputum acid fast bacilli (AFB) smear negative. Sputum culture and sensitivity yielded bacterial growth in $67 \%$ of study sample. $8 \%$ patients with fungal growth. $25 \%$ yielded no growth. Out of $67 \%$ of study sample, 29 were streptococcus pneumoniae, which was the most common organism.15 were Hemophilus influenza, 6 were Moraxella catarrhalis and 6 were Klebsiellapneumonia Spirometry (PFT) was performed in 91 patients, 9 patients were not be able to perform the test. Of the 91 studies, majority of the patients (74), had moderate airway obstruction (FEV1 between 50-80\% predicted). 2 patients had FEV1 $>/=80 \%$ predicted, 13 patients had FEV1 30-50\% predicted. Another 2 patients had FEV1 < $30 \%$ predicted. Of the total study sample, 89 patients improved and 11 patients died. In our study found that out of 11 patients died 8 had Diabetes Mellitus. The study is statistically highly significant. ( $p=0.002$ ). Our study shows that out of 11 patients died 4 had CAD. The ' $p$ ' value obtained is not statistically significant.

Out of 32 patients with Diabetes Mellitus 15 patients with Streptococcus pneumoniae on sputum culture.

Association between DM and organism cultured is statistically significant ( $\mathrm{P}$ value -0.006). In our study, out of all the patients who died, 3 patients had pseudomonas on culture and 6 patients have streptococcus pneumoniae. From our study it is clear that significant association exist between organism cultured and prognosis. This study is statistically significant. ( $\mathrm{p}$ value-0.000)

Table-1 Baseline Charecteristics

\begin{tabular}{|c|c|c|}
\hline & NUMBER & PERCENTAGE \\
\hline \multicolumn{3}{|l|}{ AGE } \\
\hline $40-49$ & 6 & $6 \%$ \\
\hline $50-59$ & 20 & $20 \%$ \\
\hline $60-69$ & 42 & $40 \%$ \\
\hline$>70$ & 32 & $32 \%$ \\
\hline \multicolumn{3}{|l|}{ SEX } \\
\hline MALE & 97 & $97 \%$ \\
\hline FEMALE & 3 & $3 \%$ \\
\hline \multicolumn{3}{|l|}{ OCCUPATION } \\
\hline NOT WORKING & 3 & $3 \%$ \\
\hline UNSKILLED & 53 & $53 \%$ \\
\hline SKILLED & 30 & $30 \%$ \\
\hline BUISENESS & 14 & $14 \%$ \\
\hline \multicolumn{3}{|l|}{ TYPE SMOKING } \\
\hline BEEDI & 67 & $67 \%$ \\
\hline CIGARETTES & 33 & $33 \%$ \\
\hline \multicolumn{3}{|l|}{ PACK YEARS } \\
\hline$>20$ & 29 & $29 \%$ \\
\hline$<20$ & 71 & $71 \%$ \\
\hline \multicolumn{3}{|l|}{ COMORBIDITIES } \\
\hline DIABETES MELLITUS & 32 & $32 \%$ \\
\hline HYPERTENSION & 26 & $26 \%$ \\
\hline $\begin{array}{c}\text { CORONARY ARTERY } \\
\text { DISEASE }\end{array}$ & 29 & $29 \%$ \\
\hline NIL & 13 & $13 \%$ \\
\hline
\end{tabular}


Table-2 Symptom Analysis

\begin{tabular}{|c|c|c|}
\hline SYMPTOMS & FREQUENCY & $\%$ \\
\hline $\mathrm{COUGH}$ & & \\
\hline YES & 100 & 100 \\
\hline NO & 0 & 0 \\
\hline EXPECTORATION & & \\
\hline SCANTY MUCOID & 6 & 6 \\
\hline $\begin{array}{l}\text { INCREASED } \\
\text { PURULENCE }\end{array}$ & 94 & 94 \\
\hline BREATHLESSNESS & & \\
\hline YES & 100 & 100 \\
\hline NO & 0 & 0 \\
\hline CHEST PAIN & & \\
\hline YES & 9 & 9 \\
\hline NO & 91 & 91 \\
\hline HEMOPTYSIS & & \\
\hline YES & 6 & 6 \\
\hline $\mathrm{NO}$ & 94 & 94 \\
\hline FEVER & & \\
\hline YES & 47 & 47 \\
\hline $\mathrm{NO}$ & 53 & 53 \\
\hline
\end{tabular}

Table -3 Comparison of DM based on outcome

\begin{tabular}{|c|c|c|c|c|c|c|}
\hline \multirow{2}{*}{$\mathrm{DM}$} & \multicolumn{2}{|c|}{ Improved } & \multicolumn{2}{|r|}{ Died } & \multirow[t]{2}{*}{2} & \multirow[t]{2}{*}{$\mathrm{P}$} \\
\hline & Count & Percent & Count & Percent & & \\
\hline No & 65 & 95.6 & 3 & 4.4 & \multirow[t]{2}{*}{$9.42 * *$} & \multirow[t]{2}{*}{0.002} \\
\hline Yes & 24 & 75.0 & 8 & 25.0 & & \\
\hline
\end{tabular}

Table 4 Comparison of DM and organism cultured

\begin{tabular}{|c|c|c|c|c|c|c|}
\hline \multirow{2}{*}{ Sputum C\&S } & \multicolumn{3}{|c|}{ DM } & \multirow{2}{*}{ Yes } & & \multirow{2}{*}{$\mathrm{p}$} \\
\cline { 2 - 5 } & \multicolumn{3}{|c|}{ No } & & \\
\cline { 2 - 5 } & Count & Percent & Count & Percent & & \\
\hline No growth & 23 & 33.8 & 2 & 6.3 & & \\
\hline $\begin{array}{c}\text { Streptococcus } \\
\text { pneumonia }\end{array}$ & 14 & 20.6 & 15 & 46.9 & \multirow{2}{*}{0.006} \\
\hline $\begin{array}{c}\text { Hemophilus } \\
\text { influenza }\end{array}$ & 9 & 13.2 & 6 & 18.8 & \\
\hline Pseudomonas & 1 & 1.5 & 2 & 6.3 & \\
\hline Others & 21 & 30.9 & 7 & 21.9 & \\
\hline
\end{tabular}

**: - Significant at 0.01 level

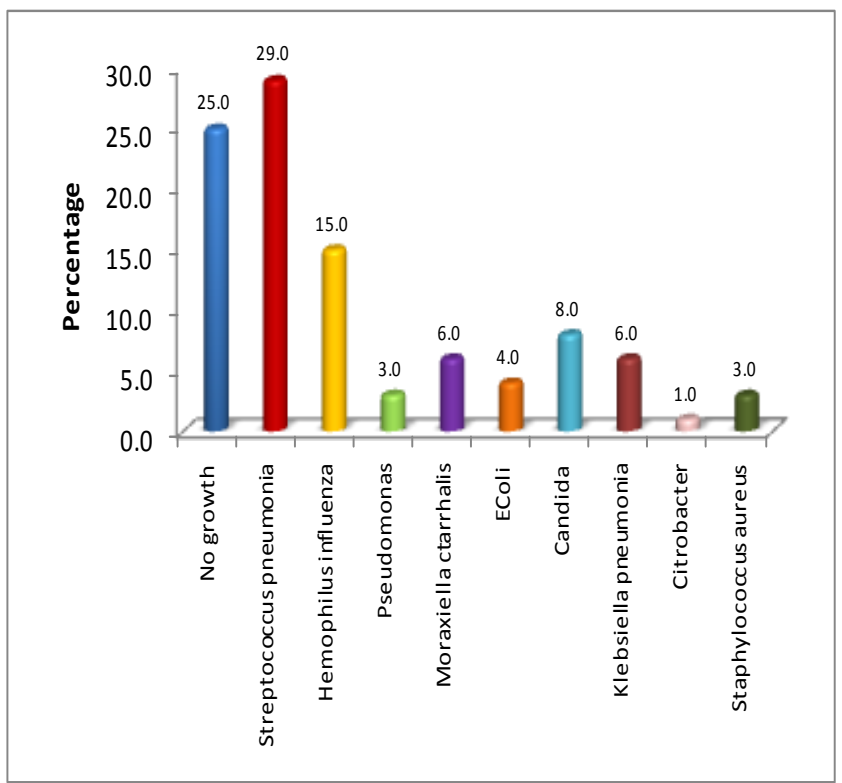

Fig.1 Percentage distribution of the sample according to sputum $\mathrm{C} \& \mathrm{~S}$

\section{COPD SEVERITY ACCORDING TO PFT}

$\square$ MILD $\backsim$ MODERATE $\backsim$ SEVERE $\backsim$ VERY.SEVERE

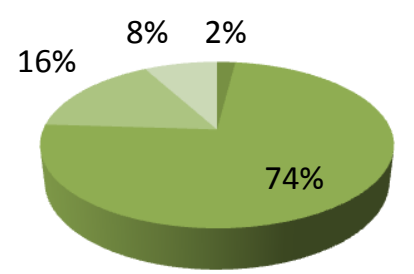

Fig-2 


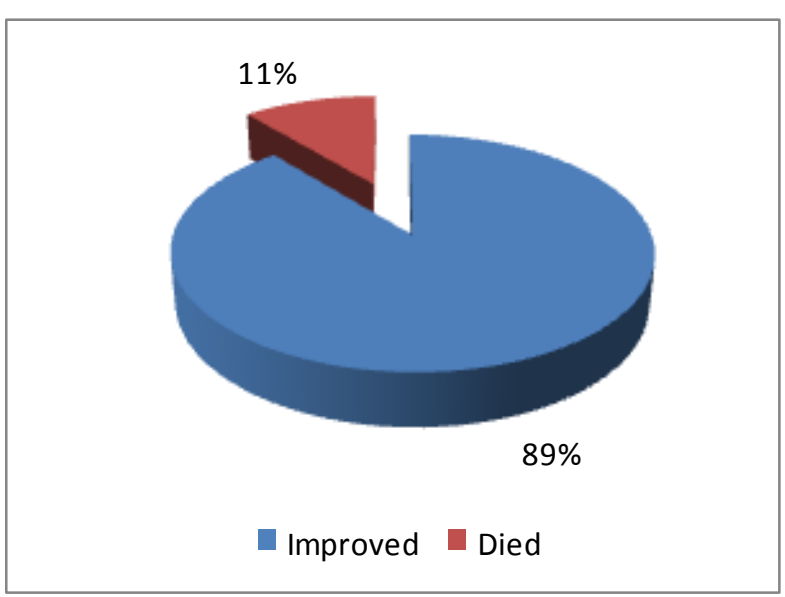

Fig. 3 Percentage distribution of the sample according to outcome

\section{DISCUSSION}

In the present study most common age group was 60-69 years. Arora et al ${ }^{5}$ studied forty eight patients with acute exacerbation of COPD (AECOPD). In that study most patients were in the age group 50-60 years .Based on Micheal et $\mathrm{al}^{6}$ study, on patients with AECOPD, the observed mean age was $68+/-9$ years. According to Robert et al ${ }^{7}$ study, which consisted 1400 patients, it was observed that more than $75 \%$ of the patients were more than 65 years old. Study of Tsai et al ${ }^{8}$ showed older patients had more attacks of AECOPD.

In our current study, most of the patients were from the elderly age group. Hence, our study correlates with previous studies. From the above studies, we can infer that COPD is a disease that occurs later in life.

In our study $97 \%$ patients were males. Only 3\% patients were females. Arora et al ${ }^{5}$ studied that $67.2 \%$ males and $32.76 \%$ were females. According to Robert et al ${ }^{7}$ among 1373 patients $54 \%$ were males and $46 \%$ were females. Our findings go in hand with other studies. This gender difference is predominantly due to greater prevalence of smoking among males. In the present study all patients were smokers. Out of this $67 \%$ patients were beedi smokers and 33\% were cigarette smokers Tsai et $\mathrm{al}^{8}$ studied that higher number of pack years of smoking is a good predictor for readmission. Gunenet $\mathrm{al}^{9}$ studied 205 patients with AECOPD. Among them $86.82 \%$ had significant pack years. Only a small percent of people were nonsmokers. According to our study, all the patients presented with cough and breathlessness. Most of the patients presented with increased sputum volume or purulence. Roberts et $\mathrm{al}^{8}$ studied 1342 COPD patients and observed that the presence of pedal edema as one of the most significant predictor of death. Connor's et $\mathrm{al}^{10}$ in their prospective study of 1016 patients with AECOPD observed similar clinical finding.

In our study, $87 \%$ patients were associated with one or more co-morbid illness. Out of which Diabetes mellitus was commonest (36.7\%). Coronary artery disease was present in 29 patients (33.33\%). Hypertension was present in 26 patients (29.8). Antenolli et al ${ }^{11}$ studied 270 COPD patients and among them hypertension (28\%) was the most common co-morbid illness. Diabetes and coronary artery disease were also common in the study group. Michael et $\mathrm{al}^{6}$ studied 39 AECOPD patients and observed that, 9patients had one or more co-morbid illness. According to Cazola et al ${ }^{12}$ study patients were at increased risk for cardiovascular events (37.4\%). Our study findings co-related with the findings of other studies.In the present study out of 100 patients, 47 patients showed abnormal Chest X-ray (parenchymal infiltrates) other than COPD changes. A study by Mohan et $\mathrm{al}^{13}$ observed presence of infiltrates in 41.4\% patients with AECOPD which almost correlates with our study. Tsai et $\mathrm{al}^{8}$ studied 384 COPD patients and observed the presence of newinfiltrates in $21 \%$ of patients. Chest X-ray is a routine screening investigation for those patients with AECOPD, not only to diagnose the cause of exacerbation, but also to exclude other differential diagnosis of AECOPD In the present study of 100 patients, causative organisms were isolated in $75 \%$ of patients.

The most common organism isolated was Streptococcus pneumoniae. In our study sputum culture showed significant bacterial growth in $67 \%$ of cases. Sethiet $\mathrm{al}^{14}$ studied AECOPD patients and observed infection of the distal airways in $50 \%$ of the patients. Hemophilus influenza, Streptococcus pneumoniae and 
Moraxella catarrhalis were the predominant bacteria isolated. Fisher et $\mathrm{al}^{15}$ studied and found more potentially pathogenic organisms in particular, Pneumococci and $\mathrm{H}$ influenza and viral and mycoplasma organism in patients during exacerbations state. Sherk $\mathrm{P} \mathrm{A}^{16}$ concluded that $50-70 \%$ of the isolates were bacterial and $20-30 \%$ viral isolates. Arora et al ${ }^{5}$ studied 58 patients with AECOPD and the following organisms were isolated-streptococcus, pseudomonas, Klebsiella and staphylococci.I.S Patel et $\mathrm{al}^{17}$ studied 29 patients with COPD exacerbations and found that $51.7 \%$ yielded a positive culture of one or more potentially pathogenic microorganism include H.influenza (53.3\%),S. pneumoniae $33.3 \%$, and M.catarrhalis. Nester Soleret al ${ }^{18}$ study found $72 \%$ patients had potentially pathogenic organisms an $12 \%$ had no pathogens in any sample. Of that $56 \%$ of pathogens were S.pneumoniae, H.influenza and M.catarrhalis.

\begin{tabular}{|c|c|}
\hline Study & \% of organism cultured \\
\hline Present study & S.pneumoniae(29\%) \\
& H.influenza (15\%) \\
& Moraxella catarrhalis(6\%) \\
Umut S etal & S.pneumoniae-12\% \\
& H.influenza-30.8\% \\
& Moraxella catarrhalis-7.7\% \\
I S Patel et al & S.pneumoniae -33.3\% \\
& H influenza-53.3\% \\
\hline
\end{tabular}

According to Hirschmann ${ }^{20}$, in his study stated that bacterial pathogens such as S.pneumoniae and H.influenza are absent in about $50 \%$ of acute exacerbations of COPD. This is against our study. He concluded that antibiotics confer no advantage for acute exacerbations of COPD. Based on Aroraet alstudy ${ }^{5}$ at Delhi, showed bacteria were cultured in $52 \%$ cases. Most common organism was streptococcus pneumoniae $(25.8 \%)$. Pseudomonas aeruginosa (12\%), Klebsiella (10.5\%), staphylococcus aureus (1.7\%). Hemophilus influenza was not isolated in any case.

In the present study out of 100 patients 89 improved and11 died. Comorbidities have a significant role on prognosis. Out of the 11 patients died some of them had one or more of the comorbid conditions. Out of 11 patients died 8 had Diabetes mellitus. Sputum culture and sensitivity in diabetic patients showed out of 8 patients died 3 of them had pseudomonas in sputum. In our study association between sputum culture and Diabetes Mellitus is statistically significant.

Our study found that 3 patients with pseudomonas on sputum culture died.6 patients out of 23 with streptococcus pneumoniae died. This study is statisticallysignificant.Miravitillis ${ }^{21}$ sudy found that more severely affected patients showing a greater incidence of pseudomonas.

In our study 29 patients had cardiovascular diseases. Based on severity of COPD 22 patients were in moderate obstructive COPD. Sin DD et al study showed that cardiovascular illness is the major reason of death in patients with mild to moderate COPD. ${ }^{22}$ According to previous studies FEV1 is a strong predictor of mortality in cardiac diseases $^{23}$.For every $10 \%$ decrease in lung function (as measured by forced expiratory volume in $1 \mathrm{~s}, \mathrm{FEV}_{1}$ ) cardiovascular mortality increases by nearly $30 \%$. Adams $\mathrm{SG}^{24}$ in his study concluded that COPD is complicated by frequent and recurring acute exacerbations and that high risk patients includes patients with co-morbid illness like diabetes mellitus, coronary artery disease etc. But according to our study severity of COPD is not associated with cardiovascular risk. This may be due to small size of population studied.

\section{CONCLUSION}

COPD and its exacerbations are a clinically significant illness in Malabar area of Kerala leading to major morbidity and mortality. In this study, most of the acute exacerbations of COPD were caused by bacterial infections. The most common organisms isolated were streptococcus pneumoniae. Co-morbid conditions like Diabetes Mellitus, Coronary artery disease and Hypertension can influence the outcome of the patients with acute infective exacerbations. Identifying the causative organisms and the anti- 
microbial sensitivity pattern will help in making a treatment protocol for the effective management of infective exacerbations of COPD. If properly treated with antibiotics according to the sputum culture and sensitivity report, mortality and morbidity can be minimized in patients with acute exacerbations of COPD. Also there needs to have strict control over associated co-morbidities which significantly affects the outcome.

Limitation: Single centered study.

Funding: No funding sources

Conflict of interest: None declared

Ethical approval: The study was approved by the Institutional Ethics Committee

\section{REFERENCE}

1. Mathers CD, LoncarD.Projections of global mortality and burden of disease from 2002 to2030.PLoS Med2006;3; e442.

2. E F M Wouters.Chronic obstructive pulmonary disease.5:Systemic effects of COPD.Thorax 2002;57:1067-1070.

3. Centers for Disease Control and Prevention(CDC).COPD among adults United States, 2011.MMWK.Morb Mortal Wkly Rep 2012;61;938.

4. Minino A M,Murphy S L,XuJ, Kochanek KD.Deaths:final data for2008.Natl Vital Stat Rep 2011;59:1.

5. Arora N, Daga MK, Mahajan R, Prakash SK, Gupta N. Microbial pattern of acute infective exacerbation of chronic obstructive airway disease in a hospital based study. Indian J Chest Dis Allied Sci 2001; 43: 157-62

6. Michael et al.Predictors of outcome for patients with COPD requiring invasive mechanical ventilation.Chest 2001; 119: 1840-9.

7. Roberts CM, Lowe D, Bucknall CE, Ryland I, Kelly Y, Pearson MG.Clinical audit indicators of outcome following admission to hospital with acute exacerbation of chronic obstructive pulmonary disease. Thorax 2002; 57: 137.
8. Tsai et al.Factors associated with hospital admission among emergency department patients with chronic obstructive pulmonary disease exacerbation.Academic Emergency Medicine 2007;14:6-15

9. Gunen $\mathrm{H}$ et al.Factors affecting survival of hospitalised patients with COPD .EurRespir J 2005;26:234-41

10. Connorsetal.Outcomes following acute exacerbation of severe chronic obstructive lungdisease.The SUPPORT investigators (Study to understand prognoses and preferences for outomes and risks of treatments.)Am J RespirCrit Care Med1996;154:959-67.

11. Antenolli et al.Co -morbidity contributes to predict mortality of patients with chronic obstructive pulmonary disease. Eur Respir J1997;10:2794-800

12. Cazola et al.Prevalence of comorbidities in patients with Chronic obstrucive pulmonary Disease .Respiration 2010;80:112-119.

13. Mohan A, Premanand R, Reddy LN, Rao MH, Sharma SK, Kamity R et al.Clinical presentation and predictors of outcome in patients with severe acute exacerbation of chronic obstructive pulmonary disease requiring admission to intensive care unit. BMC Pulmonary Medicine 2006; 6: 27.doi10.1186/1471-2466-6-27

14. SanjaySethiMD.Infectiousetiology of acute exacerbtions of chronic bronchitis. Chest 2000;117:380S-385S.

15. Fisher M,AkhtarAJ,Calder M A,etal.Pilot study of factors associated with exacerbations in chronic bronchitis. BMJ1 969;4:187-192.

16. Sherk P A , Grossman RF. The chronic obstructive pulmonary disease. Clinics in Chest Medicine.21:705-721,2000

17. S ,Pateletal. Relationship between bacterial colonization and the frequency, character and severity of COPD exacerbation. Thorax 2002;57:759-764. 
18. Nestor Soleretal. Bronchial microbial pattern in severe exacerbations of chronic obstructive pulmonary disease requiring mechanical ventilation. Amj Respir. Crit.Care Med.1998;157:1498-1505

19. Umut $S$ et al. Determination of the etiological organism during acute exacerbations of COPD and efficacy of azithromycin, ampicillinsulbactam, ciprofloxacin and cefaclor. Turkish Thoracic Society COPD Working Group. Journal of chemotherapy 1999;211-214.

20. HirschmannJV. Do bacteria causes exacerbations of COPD? Chest 2000;118:193-203

21. Marc Miravitllesetal. Relationship between bacterial flora in sputum and functional impairment in patients with acute exacerbations of COPD. Chest 1999;116:40-46

22. Sin DD, Anthonisen NR, Soriano JB, AgustiAG.Mortality in COPD: role of comorbidities. EurRespir J2006; 28(6): 1245-57

23. Iversen KK, Kjaergaard J, Akkan D, et al. The prognostic importance of lung function in patients admitted with heart failure. Eur J Heart Fail 2010;12:685-91

24. Adams S G, Anzeuto A. Antibiotic therapy in acute exacerbations of chronic bronchitis. Seminars in respiratory infections.2000;15:234-237 